\title{
Acerca de una experiencia de formación docente fundada en la didáctica de géneros: configuración del objeto de enseñanza e interacción pedagógica
}

\author{
On a teacher training experience in genre-based pedagogy: configuration of the object of teaching and \\ guided interaction
}

\author{
María Lorena Bassa Figueredo (D) \\ Universidad Nacional de General Sarmiento - UNGS - Buenos Aires - Argentina
}

Estela Ines Moyano (D)

Universidad Nacional de General Sarmiento - UNGS - Buenos Aires - Argentina

\begin{abstract}
Resumen: La propuesta didáctica basada en géneros desarrollada por la llamada Escuela de Sídney (MARTIN, 1999, 2009; MARTIN; ROSE, 2005, 2008; ROSE; MARTIN, 2012, entre otros) y su reformulación argentina (MOYANO 2007, 2013) apuntan a diseñar una didáctica de la lectura y la escritura que permita a cualquier estudiante satisfacer las demandas escolares. Es por esto que se considera valiosa también en la formación docente de profesores de Lengua y Literatura. Así, en el Profesorado en Lengua y Literatura la Universidad Nacional de General Sarmiento, de Argentina se ha optado por esta propuesta en sus dos asignaturas de didáctica específica. En particular, en este artículo interesa dar cuenta de una experiencia formativa desarrollada a partir del segundo semestre de 2018 y durante 2019. Con este fin, se propone analizar las secuencias didácticas (SD) elaboradas por 13 estudiantes en ese período. Para el análisis, se construyen cinco categorías conceptuales que se integran, a su vez, en dos dimensiones que permiten exponer y discutir los hallazgos. Este análisis diacrónico de las SD posibilita evaluar un proceso formativo en relación con el grado de apropiación de la propuesta didáctica que se logra alcanzar.
\end{abstract}

Palabras Clave: didáctica de géneros; formación docente; objeto de enseñanza; interacción pedagógica

\begin{abstract}
The genre-based pedagogic proposal developed by the so-called Sydney School (MARTIN, 1999, 2009; MARTIN; ROSE, 2005, 2008; ROSE; MARTIN, 2012, among others) has been adapted in Argentina (MOYANO 2007, 2013). These proposals aim to support all students to satisfy the school requirements through processes of teaching and learning reading and writing abilities. Consequently, they are appraised resources to train future Language secondary teachers. This option has been taken in the two subjects devoted to teaching specific pedagogic proposals at the Profesorado en Lengua y Literatura at the Universidad Nacional de General Sarmiento, in Argentina. The purpose of this paper is to give account of an experience that took place in this context from August 2018 to December 2019. To achieve this purpose, the pedagogic sequences elaborated by 13 university students has been analysed. In order to do this, five conceptual categories integrated in two dimensions have been designed. They have allowed to present and discuss the results obtained. This analysis showed a teaching-learning process related to the appropriation of the genre-based proposal by the future teachers.
\end{abstract}

Keywords: genre-based pedagogy; teacher training; object of teaching; guided interaction 


\section{INTRODUÇÃO}

La didáctica de géneros desarrollada por la llamada Escuela de Sídney (MARTIN, 1999, 2009; MARTIN; ROSE, 2005, 2008; ROSE; MARTIN, 2012, entre otros) y su reformulación argentina (MOYANO, $2007,2013)$ apuntan a favorecer el desarrollo del lenguaje $y$, en particular, de aquellas formas del lenguaje propias de esferas institucionales educativas que habilitan el acceso a la construcción de conocimientos diferentes a los del sentido común, en particular, los géneros escolares y científicos. De ahí la importancia de enseñarla, junto con los géneros discursivos escolares, en el marco de la formación docente de profesores de Lengua y Literatura.

En efecto, en el Profesorado en Lengua y Literatura de la Universidad Nacional de General Sarmiento, em Argentina se ha optado por la formación docente en la propuesta didáctica centrada en géneros y basada en la Lingüística SistémicoFuncional (LSF) adaptada por Moyano (2007, 2013). Este trabajo se realiza en el área de Lengua de dos asignaturas correlativas de didáctica específica: Enseñanza de la Lengua y la Literatura (semestral) y Residencia II en Lengua y Literatura (anual).

En particular, en este artículo interesa dar cuenta de la experiencia formativa de esas dos asignaturas durante el segundo semestre de 2018 (Enseñanza de la Lengua) y el año calendario 2019 (Residencia II en Lengua). Para esto, se propone analizar las secuencias didácticas (SD, en adelante) elaboradas por estudiantes a lo largo de ese año y medio de formación. Se entiende que este análisis diacrónico de las SD posibilita ponderar un proceso formativo en relación con el grado de apropiación de la propuesta didáctica que los docentes en formación logran alcanzar.

En primer lugar, se da cuenta del marco conceptual que fundamenta la propuesta didáctica. En segundo lugar, se explicita la metodología con la que se estudian las SD y el procedimiento con el que se construyen las cinco categorías conceptuales y las dos dimensiones analíticas. A continuación, se presentan los resultados de la indagación siguiendo las categorías propuestas. En cuarto lugar, se discuten las dimensiones indagadas en relación con conceptos teóricos pertinentes y se consideran prospectivamente intervenciones en la formación docente. Por último, se resumen los hallazgos más relevantes del análisis y su posible contribución a la formación de docentes de Lengua / Prácticas del Lenguaje.

\section{Marco teórico}

La propuesta didáctica elaborada por Moyano $(2007,2013)$ es el marco que orienta el trabajo que se solicita a los estudiantes del Profesorado en el período de formación en las didácticas específicas. Esta propuesta, como ya se señaló, es una adaptación del Ciclo de Enseñanza Aprendizaje elaborado por Martin y sus discípulos (MARTIN, 1999, 2009) así como de la didáctica denominada Leer para Aprender (R2L por su nombre en inglés) (ROSE; MARTIN, 2012).

En la Figura 1 se sintetiza el diseño didáctico adoptado. Como se puede observar, consta de tres etapas: Deconstrucción del género a trabajar, Construcción de un texto que instancie el género y Edición del texto elaborado. La segunda etapa está precedida por una subetapa, el Diseño del texto, que permite a los estudiantes reflexionar sobre la organización del escrito a producir antes de hacerlo. Estas tres etapas están atravezadas por la reflexión sobre el registro, en particular sobre el campo, así como también por una consideración acerca del género y las diferentes opciones entre las que se seleccionan el que se va a producir. Por otro lado, las tres etapas se realizan teniendo en cuenta un proceso que va desde la heteronomía a la autonomía: se trabaja primero en conjunto entre el grupo de estudiantes y el docente, en un contexto de interacción compartida; en segundo lugar en pequeños grupos y finalmente en un trabajo individual, autónomo, que solo puede hacerse luego de las experiencias anteriores. 


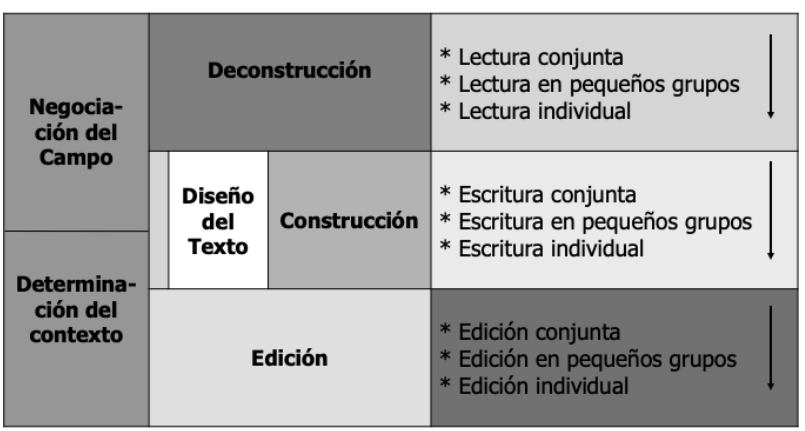

Figura 1. Propuesta didáctica basada en géneros aplicada al contexto de formación de futuros docentes (MOYANO, 2007, 2013)

La primera etapa, Deconstrucción, consiste en el análisis conjunto entre docentes y estudiantes de un texto como instancia del género que se pretende abordar. Esta tarea se orienta a la identificación de su propósito social, la estructura esquemática mediante la cual se logra ese propósito y la identificación de los principales recursos lingüísticos mediante los cuales se construye significado en el texto. Estos recursos son sistematizados, de manera de avanzar en su aprendizaje a fin de que los estudiantes se los apropien, por un lado, como conocimiento de los diferentes sistemas de lenguaje así como también para aplicarlos en la producción de nuevos textos. Se procura centrar estas reflexiones en los sistemas del estrato semánticodiscursivo, aunque también se abordan, cuando es necesario, recursos del estrato léxicogramatical (MARTIN; ROSE, 2007).

La etapa Construcción de un nuevo texto como instancia del género en estudio se lleva a cabo, en primera instancia, de manera conjunta. Para ello, el docente provee de materiales como infografías, tablas, líneas de tiempo u otros recursos para ofrecer contenido sobre el cual trabajar. A partir de allí, guía a la clase para el Diseño del texto, que se hace teniendo en cuenta los mismos recursos que en la Deconstrucción, utilizando esquemas para la organización del nuevo texto. Luego, mediante diferentes recursos didácticos, como pizarrón, afiches - sistemas electrónicos, si se pudiera contar con ellos, docente y estudiantes escriben conjuntamente a partir del diseño elaborado. Nuevamente, se presta atención al propósito del texto, su estructura esquemática y los recursos relevantes para la construcción de significado. A partir de allí, se inicia una etapa de Construcción de un nuevo texto, esta vez de manera grupal o individual, a partir de nuevos materiales ofrecidos por el docente.

Finalmente, la tercera etapa consiste en enseñar a los estudiantes a llevar a cabo el trabajo de Edición, tarea que llevan a cabo los escritores expertos luego de finalizar la primera versión de su texto. Para ello, se guía mediante diferentes recursos la observación de los trabajos elaborados, teniendo en cuenta si presentan las características que fueron enseñadas en las etapas anteriores y si producen significado con claridad. Para ello, el docente modela la tarea, tal como hizo en las etapas anteriores, comenzando con la Edición conjunta de un par de textos producidos por los estudiantes. A partir de allí, supervisa el trabajo que se realiza en pequeños grupos o individualmente.

Para realizar cada una de las etapas de manera conjunta, el docente tiene que tener en cuenta la preparación de los estudiantes para dar respuesta a las preguntas mediante las cuales guía el trabajo. Para ello, se sigue la propuesta de Rose y Martin (2012), que sostiene para cada tarea una secuencia de cinco etapas: Preparación, Foco, Tarea, Elaboración, Evaluación (Figura 2).

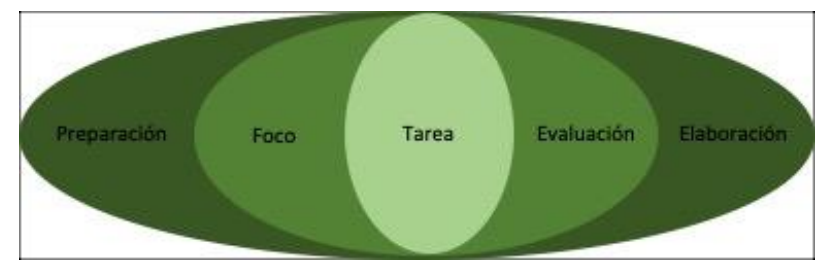

Figura 2. Estructura nuclear de las actividades de aprendizaje (ROSE; MARTIN, 2012)

Este tipo de secuencia orienta al estudiante a la hora de efectuar la actividad que se le solicita y lo 76 uia para hacerlo exitosamente. Esto permite que los estudiantes más desaventajados tengan oportunidad frente a los de códigos más elaborados (Bernstein, 1990), que son los que habitualmente participan de la clase y obtienen los mejores resultados. Se trata de preparar a los estudiantes para dar respuestas a las preguntas-guía de los docentes, de manera que puedan hacerlo 
adecuadamente, recibir confirmación y así obtener la confianza que necesitan para desarrollar exitosamente sus aprendizajes (MARTIN; ROSE, 2005). Luego de cada ciclo conformado por los pasos de la estructura nuclear de actividades de aprendizaje se inicia uno nuevo, de manera que la clase se desarrolla así uma uma 77uia efectiva para todos los estudiantes y se contribuye a acortar la brecha entre los más y menos aventajados.

\section{Marco metodológico}

Esta comunicación se propone analizar las variaciones diacrónicas de las secuencias didácticas (SD) para la enseñanza de la Lengua elaboradas por quienes realizaron sus prácticas docentes en 2019 en el Profesorado Universitario de Educación Superior en Lengua y Literatura de una universidad argentina. En este sentido, este estudio combina lo descriptivo y lo exploratorio, dado que se propone no sólo caracterizar algunas dimensiones de estos documentos elaborados por los estudiantes sino también interpretar los distintos grados de apropiación de la propuesta didáctica que implican. Por este alcance hermenéutico es que se asume un enfoque cualitativo de fuerte orientación interpretativa (SAUTU, 2005). El análisis de las SD realizadas en el lapso considerado permite evaluar la evolución de un proceso educativo de docentes en formación.

\section{$2.1 \quad$ Corpus}

El corpus está conformado por las secuencias didácticas (SD) elaboradas para el área de Lengua por los residentes tanto en la asignatura de Enseñanza de la Lengua y la Literatura en 2018 como aquellas diseñadas por los mismos estudiantes durante 2019, en el marco de la asignatura anual de Residencia II en Lengua y Literatura. Estas últimas fueron dos: una en el primer semestre (antes de las prácticas docentes) y otra en el segundo semestre, específicamente para esas prácticas áulicas.

\subsection{Diseño de investigación}

Con el objetivo de poder dar cuenta de esta experiencia, se recurre al diseño de investigación de estudio de caso. Según Sautu (2005), este diseño presenta tres rasgos distintivos: particularización, fuerte impronta descriptiva y búsqueda heurística. Lo primero se vincula con situar claramente esta experiencia puntual de formación docente en el contexto de las didácticas específicas del Profesorado Universitario de Educación Superior en Lengua y Literatura de una universidad específica entre el segundo semestre de 2018 y finales de 2019. Lo segundo supone caracterizar las numerosas secuencias didácticas que se analizan a partir de las categorías que se construyen a tal efecto y que se describen más adelante. Finalmente, como ya se expresó, se apunta a explicar didácticamente esta experiencia de formación docente a partir de la propuesta didáctica basada en géneros.

\subsection{Corpus de análisis}

Este estudio se basó en el análisis de tres instancias sucesivas de planificación de SD de una misma cohorte de 13 estudiantes, que se codifican del siguiente modo:

SD 1: Al finalizar la primera didáctica específica semestral (Enseñanza, 2do sem. 2018)

SD 2: Al comenzar la segunda didáctica específica anual (Residencia II, 1er sem. 2019)

SD 3: Al promediar la segunda didáctica específica anual (Residencia II, 2ndo sem. 2019)

Por su parte, los trece estudiantes fueron codificados alfabéticamente de la $\mathrm{A}$ a la $\mathrm{M}$.

Cada SD se centra en la enseñanza de un género, que en SD1 y SD2 fue elegido por cada estudiante. En el caso de SD3, realizada en el marco de las prácticas docentes, el género a enseñar fue negociado a partir del diálogo que se establece con el docente coformador que plantea qué contenidos va a abordar el residente en sus prácticas áulicas.

Como se trata de una secuencia didáctica basada en la propuesta descripta en el Marco Teórico 
de este artículo, cada CD está organizada en 4 clases:

Clase 1: Deconstrucción conjunta

Clase 2: Construcción conjunta

Clase 3: Construcción en parejas o individual

Clase 4: Edición conjunta y en parejas o individual

\subsection{Procedimiento}

Las SD que conforman nuestro corpus son numerosas y extensas. Por lo tanto, nuestra estrategia de análisis involucró, en primer lugar, su recolección y ordenamiento. Luego, su lectura analítica posibilitó la construcción de cinco categorías -organizadas a su vez en dos dimensiones-, que dieran cuenta de la variación diacrónica que se hipotetiza. Finalmente, se seleccionaron fragmentos representativos de esas categorías para construir nuestra muestra de análisis.

A finales de 2018, se recolectó el conjunto de SD1, producido en el marco de Enseñanza de la Lengua; en el primer semestre de 2019, el conjunto de SD2; y, en el segundo semestre el de SD3.

A continuación, en la primera lectura, se delineó una primera construcción de categorías de análisis. Para esto, se recurre al método de comparación constante (GLASER; STRAUSS, 1967), considerando el alto número de documentos recogidos. Así, se ordenaron las SD de cada estudiante en forma diacrónica y se identificaron las unidades de sentido y las categorías que daban cuenta de continuidades y variaciones a lo largo del año y medio de estudio. Las categorías fueron seleccionadas del marco teórico de referencia en función de su pertinencia para el corpus analizado. Las categorías que resultaron más relevantes para analizar las variaciones diacrónicas fueron las siguientes:

1) Géneros seleccionados para la enseñanza

2) Recursos lingüísticos abordados

3) Guion de la Deconstrucción conjunta

4) Materiales diseñados para la Construcción

5) Guion de la Edición conjunta
Estas categorías se integraron posteriormente en dos dimensiones vertebradoras de la propuesta didáctica para establecer un nivel más abstracto de análisis, tal como se verá en la Discusión. Estas dimensiones son las siguientes:

1) la configuración del objeto de enseñanza (en relación con las categorías 1 a 2) y

2) la interacción pedagógica de la propuesta didáctica (vinculada con las categorías 3 a 5).

Por último, para hacer un estudio más detallado, se construyó un muestreo configurado por 3 casos de análisis para cada categoría. Cada caso se organizó en un contraste SD1/SD2 vs. SD3. En este artículo, por una cuestión de extensión, para ilustrar cada categoría se presenta un caso, correspondiente a un residente $(A, C \circ M)$. Estos casos representan evoluciones similares a las obtenidas en los demás.

\section{Resultados}

El análisis diacrónico de las SD de cada estudiante fue realizado en función de cinco categorías que permiten dar cuenta del proceso de apropiación de la propuesta didáctica basada en géneros en la formación docente desde Enseñanza de la Lengua al segundo semestre de Residencia II en 2019. A continuación, se muestran los resultados del análisis para cada una de ellas.

\subsection{Géneros discursivos seleccionados para la enseñanza}

En relación con la selección de géneros, en el conjunto de SD1 predominan géneros como la Biografía, el Relato Histórico y distintos tipos de Informes, es decir, géneros de menor complejidad. Por el contrario, en SD3, se amplía el abanico de géneros enseñados y se planifican secuencias para abordar también aquellos organizados por relaciones causales tanto externas (Explicación de consecuencias) como internas (géneros persuasivos). Así, en esta experiencia se observa que los docentes en formación en sus planificaciones sucesivas siguen 
también el mismo trayecto de aprendizaje de géneros que propone el marco teórico para los estudiantes (COFFIN, 1997; MARTIN; ROSE, 2008). Es decir, en una primera aproximación a la propuesta didáctica, seleccionan trabajar con géneros organizados temporalmente para luego avanzar hacia el abordaje de géneros organizados por relaciones causales, que ponen en juego recursos lingüísticos de mayor abstracción.

Respecto de las fuentes de las que se obtienen los textos modélicos que se usan en la Deconstrucción, en SD1 y SD2, observamos que se circunscriben principalmente a manuales de secundaria de editoriales grandes (Santillana y Aique, por ejemplo) aunque también, en menor medida, de editoriales independientes (como Estación Mandioca y Tinta Fresca). En SD3, en contraste, se incorporan textos de otros ámbitos, como los periodísticos o los de divulgación. De este modo, lo que en las primeras planificaciones se limita más bien a textos didácticos de manuales va ampliándose a otros ámbitos más vinculados a ciertas discusiones sociales contemporáneas.

\subsection{Recursos lingüísticos enseñados}

Las SD seleccionan los recursos lingüísticos más relevantes para construir significado en el género abordado y en función del año para el que se planifica. En esa selección se tiene en cuenta también la relación de esos recursos con los ya trabajados anteriormente con los estudiantes secundarios.

En la Deconstrucción se analizan esos recursos en el texto que se toma como "modelo" del género a trabajar; en la Construcción, se ponen en juego para escribir un nuevo texto de ese mismo género $y$, finalmente, en la Edición, se reflexiona acerca su uso adecuado o inadecuado, a fin de lograr la elaboración de una versión definitiva del texto. Por esto es que resulta fundamental seleccionar criteriosamente los recursos que se enseñarán en las tres etapas de la propuesta en función de que sean significativos para que los estudiantes puedan leer, escribir y editar el género.
A partir del análisis diacrónico de las planificaciones, resultaron notables las diferencias en la selección de recursos lingüísticos entre SD1 y SD2, por un lado, y SD3, por otro. El siguiente caso es elocuente en este sentido.

Como se observa en el Ejemplo 1, la estudiante C plantea en su SD2 para Relato Histórico la enseñanza de tipos de procesos y tipos de participantes. Es decir, selecciona recursos del estrato léxicogramatical y lo hace con un enfoque tipológico.

\section{Ejemplo 1}

Recursos lingüísticos:

-los procesos materiales, relacionales y mentales;

-los participantes genéricos y específicos [SD2, C]

Posteriormente, la misma estudiante, en su SD3, en la que enseña el género Explicación Secuencial, propone abordar los sistemas de conjunción y de periodicidad del estrato semánticodiscursivo (MARTIN; ROSE, 2007).

\section{Ejemplo 2 \\ Recursos lingüísticos: \\ -relaciones lógicas (conjunciones explícitas e implícitas, metáfora gramatical lógica) \\ -momentos de anticipación o síntesis (relación con las etapas del género) [SD3, C]}

Como se evidencia en este pasaje, en SD2 se seleccionan recursos del estrato léxicogramatical, mientras que en SD3 se trabaja con sistemas del estrato semánticodiscursivo, que implican un abordaje más abstracto y de un alcance más extenso del texto (MARTIN; ROSE, 2007). Estos últimos, además, se entienden vinculados a la estructura del género: en la Explicación Secuencial el primer paso (Fenómeno) se realiza en un macroTema (periodicidad) y se establecen entre pasos relaciones lógicas causales (conjunción) propias del género. De este modo, se evidencia que en SD3 la docente en formación planea enseñar recursos que reflexionan y andamian aspectos más globales de los textos y se alejan del alcance más local y clasificatorio que se propone en SD2 (donde tanto procesos como participantes se 
piensan taxonómicamente pero sin tener claro el objetivo didáctico de esa disquisición).

\subsection{Guion de la Deconstrucción conjunta}

La etapa de la Deconstrucción Conjunta se realiza mediante interacciones guiadas entre docente y estudiantes. El objetivo de este diálogo es caracterizar el propósito, la estructura y los recursos lingüísticos relevantes para el género trabajado, no con un afán deteccionista ni aplicacionista de la LSF sino para guiar una reflexión metadiscursiva que les permita a los estudiantes hacerse de recursos para la comprensión del texto.

Para preparar y diseñar esa interacción se les pide a los docentes en formación que escriban en sus planificaciones un guion hipotético, como forma de explicitar ese diálogo y para poder también analizarlo didácticamente.

Los siguientes ejemplos de la estudiante C sirven para ilustrar la variación que se encuentra recurrentemente entre SD1/SD2 y SD3 en estos guiones hipotéticos. En el Ejemplo 3, se presenta un fragmento de un guion hipotético para enseñar Biografía. Allí, la docente en formacón indica consignas de identificación, que solo en un principio son guionadas por la enumeración de los pasos del género.

\section{Ejemplo 3}

- Las partes de una biografía son entrada, caracterización del personaje y hechos centrales de la vida del sujeto. Identifiquen las partes en el TEXTO

-Identifiquen los verbos conjugados. En su mayoría ¿qué persona indican? ¿en singular o plural? ¿en qué tiempo verbal? ¿qué modo y aspecto indican? [SD1, C]

En su SD3, esa docente en formación elabora un guion hipotético para la Deconstrucción de una Explicación Secuencial. Como se muestra en el Ejemplo 4, el guion intercala abundantes segmentos de explicación en los que se les brinda información a los estudiantes (marcados con negrita) con consignas de identificación o con preguntas. Estas preguntas o bien presentan alternativas (que muestran las opciones del sistema lingüístico respecto del rasgo sobre el que se está focalizando), o bien dan indicios que orientan claramente su respuesta. Así, gradualmente, se va apuntalando un trabajo en detalle con el texto que involucra señalización y reflexión acerca de cómo ese paso o ese recurso construye significado.

\section{Ejemplo 4}

La Explicación secuencial en su primer párrafo orienta al lector sobre el tema. Ese paso (o etapa) se denomina fenómeno. Identifíquenla en el texto.

Luego, en los párrafos siguientes, presenta una organización temporal y causal de los eventos. Esos pasos (o etapas) se reconocen como secuencia explicativa. ¿cuántas hay? Identifíquenlas en el texto.

En el inicio de cada secuencia explicativa aparecen palabras que dan pistas del contenido del párrafo, márquenlas en cada secuencia.

Ahora, nos detenemos en el verbo "se conoce" ¿realiza una descripción (¿cómo es?) o una definición (¿qué es?)?

Marquemos los verbos, subrayen se conoce, se puede explicar, se evapora ¿están en tiempo pasado? ¿futuro? ¿o presente?

La explicación secuencial explica cómo ocurre un evento (organizado en el tiempo) en tiempo presente. Quien explica no es el protagonista de los hechos. Por eso cuenta en 3ra persona del singular o del plural. No en primera persona (ni del singular ni del plural), "yo paso/salgo/doy--Nosotros pasamos/salimos/damos". Por ejemplo: "El agua pasa a la atmósfera". Vuelvan a leer los verbos que marcaron. ¿están en 1era o en 3era persona (singular/plural)?

En la secuencia explicativa 6 (la última), los dos últimos verbos "circula" y "vuelve" ¿qué función tienen en la explicación del tema? ¿es posible vincularlos con el sustantivo "ciclo"? [SD3, C]

De este modo, el abordaje didáctico entre Ejemplo 3 y Ejemplo 4 es sumamente distinto. En el primero, se propone una tarea de detección (identificación de partes y de verbos) a partir de una exposición conceptual abstracta, que supone una operación de deducción. A su vez, se presupone el conocimiento de una serie de categorías gramaticales (persona, número, tiempo, modo y aspecto), que se dan por enseñadas previamente y que no se recuperan.

En el segundo, en cambio, se plantea un trabajo inductivo a partir de la reflexión sobre la construcción de significado en el discurso. Las preguntas que va realizando la docente, por su parte, 
se responden a partir de la lectura detallada del texto y de la "pista" explicativa brindada por la guía docente, como antesala de la respuesta. En varios casos, además, las preguntas recrean las disyuntivas del sistema lingüístico en juego, cuyo contraste es evaluado en la explicación previa. En este sentido, es que en SD3 del Ejemplo 4 se estructura la actividad de aprendizaje proveyendo la Preparación de la tarea (ROSE; MARTIN, 2012), atendiendo al andamiaje necesario para resolverla que discute la propuesta didáctica.

Vale destacar, además, que esta Preparación de la tarea es plasmada de diversos modos en distintas SD3. Al respecto, se observa que en 3 casos de las 13 SD3, se propone una actividad de sistematización de lo deconstruido conjuntamente, que podría interpretarse como una preparación para abordar los materiales diseñados para la Construcción Conjunta (Figura 3)

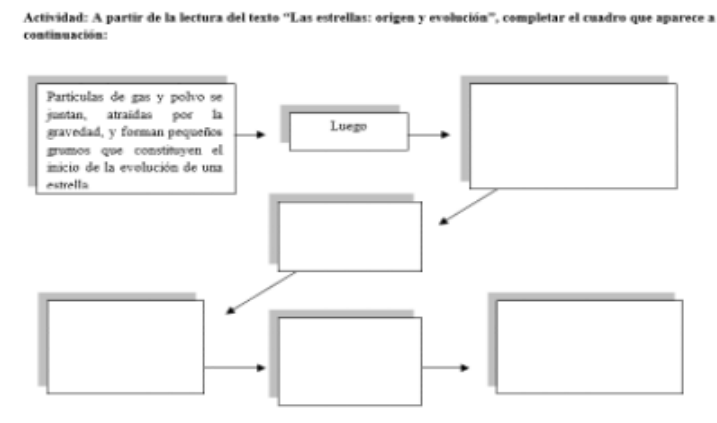

Figura 3. Materiales para la Construcción Conjunta

Así, con esta actividad, la practicante diseña un puente entre la etapa inicial de Deconstrucción y la de Construcción que le sigue, puesto que no solo plantea una sistematización de las etapas del género y su relación lógica (visto en la primera etapa) sino que prepara la lectura del material que será dado a los estudiantes para la escritura (Construcción), que tendrá un formato análogo.

\subsection{Materiales diseñados para la Construcción}

En la Construcción Conjunta, se modeliza la escritura del género que se está enseñando. Para esto la docente guía un intercambio que va a ir produciendo un texto que se construye en el pizarrón, en un afiche o en una proyección (si se tiene la posibilidad de contar con esa tecnología). Uno de los aspectos clave para planificar adecuadamente esta etapa tiene que ver con poder brindarles a los estudiantes los materiales necesarios para construir un texto nuevo.

En SD1 y SD2 se presenta como problema que los materiales que los estudiantes proponen para esta etapa son frecuentemente insuficientes. Por ejemplo, se observa que para la Construcción de una Biografía o un Relato Histórico muchas veces el insumo que se les ofrece a los estudiantes es una línea del tiempo que se toma de alguna fuente publicada. Sin embargo, este material no presenta toda la información necesaria, por ejemplo, para construir el primer paso de ambos géneros (Presentación / Orientación, respectivamente) o el último de ambos (Evaluación).

A diferencia de estos materiales insuficientes, en DS3 se encuentra, en la amplia mayoría de los casos, una propuesta más genuina de escritura que se plantea a partir de materiales elaborados ad hoc con la totalidad de la información necesaria para producir un nuevo ejemplar del género.

La Figura 4 muestra un material elaborado por una docente en formación (M) para construir una Explicación Secuencial sobre formación de estrellas. La residente ha elaborado este cuadro a partir de un texto del género y mantiene en el gráfico un código que ya trabajó con los estudiantes mediante el cual se representan las relaciones lógicas con unas flechas que apuntan hacia las consecuencias.

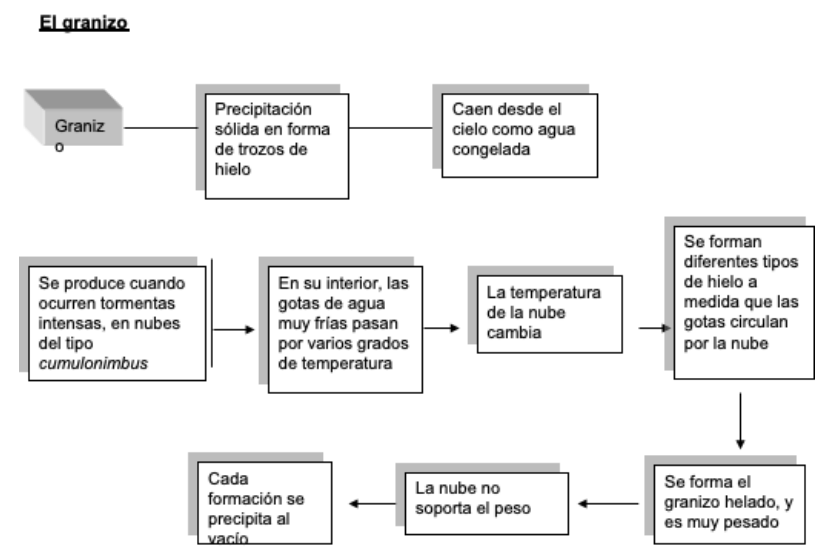

Figura 4. Material diseñado para la Construcción Conjunta de una Explicación Secuencial [SD3, M] 
Este material brinda, efectivamente, toda la información necesaria para la construcción de una Explicación Secuencial. Además, representa gráficamente las características del género en cada paso. Así, codifica con una línea la información necesaria para definir y describir el Fenómeno abordado (en el primer paso del género) y con una flecha la relación causal que articula cada uno de los pasos siguientes (Secuencias explicativas). De esta manera, la información se despliega completa y como refuerzo de la estructura esquemática del género, estableciendo una relación clara entre sus etapas y su propósito.

\subsection{Guion de la Edición conjunta}

En esta propuesta didáctica, la Edición es una etapa a ser enseñada de manera explícita y sistemática. Como este abordaje no suele formar parte de las experiencias didácticas ya conocidas por los residentes ya que "no suele ser objeto de enseñanza" (MOYANO, 2013: 117), en las primeras $\mathrm{SD}$, se evidencia que no se termina de comprender cabalmente la necesidad de modelizar cómo se edita un texto efectivamente producido por los estudiantes secundarios.

El Ejemplo 5 da cuenta de un fragmento de edición de un Relato Histórico del residente $A$ en el que este proceso toma la forma de un listado de consignas que más "tradicionalmente" apuntan a la "corrección" de los textos producidos, pero sin involucrar un andamiaje sistemático para guiar esta tarea.

Ejemplo 5

Consigna

a) Relean el texto producido la clase anterior y socialícelo con sus compañeros. Lea las producciones de sus compañeros y establezca similitudes y diferencias.

b) De forma individual, realicen en su escrito las correcciones que considere pertinentes.

c) Redacte una versión definitiva del texto que incorpore las modificaciones sugeridas durante la clase. [SD1, A]
Por el contrario, la modelización necesaria del proceso de edición sí aparece explícita en SD3 de ese mismo residente $(A)$, como se ilustra en el Ejemplo 6.

\section{Ejemplo 6}

El docente comienza la clase presentando uno o dos textos modélicos que serán expuestos en ampliaciones en papel afiche. También repartirá a los estudiantes una fotocopia con los mismos textos para que puedan ir modificándolos conjuntamente con el trabajo realizado sobre los afiches. El docente comenzará haciendo preguntas sobre qué aspectos modificarían en los textos, comenzando por el título y finalizando en el último párrafo. Se orientará a los estudiantes con preguntas para que ellos mismos sean quienes realizan los aportes para que los textos editados se acerquen más al objetivo planteado. Se revisará que esté bien presentado el problema en debate, la estructura de tesis $y$ argumentos/contraargumentos,

pidiéndoles a los estudiantes que los identifiquen, $y$ que consideren como criterio si los argumentos y el texto en su conjunto son convincentes o no. Si los contraargumentos logran neutralizar el efecto persuasivo de los primeros argumentos. Que el texto sea cohesivo y coherente, revisando el uso o la falta de conectores lógicos, de signos de puntuación y que se eviten las repeticiones de palabras. Que la tesis sea correctamente reformulada al final del texto.

Luego de intervenir conjuntamente el o los textos modélicos, los estudiantes, en parejas, harán lo propio con los textos escritos por ellos mismos la clase anterior. Se les pedirá que no borren nada en el texto original sino que tachen, usen colores y le realicen anotaciones en los márgenes. Luego, lo pasarán en limpio. Al finalizar la clase serán entregados al docente ambos escritos: el primer borrador con las correcciones hechas y una segunda hoja con el texto final, a fin de evaluar las transformaciones introducidas en los textos. [SD3, A]

En este último ejemplo, se despliega más claramente en qué consiste el andamiaje necesario para realizar la Edición conjunta del género. En primer lugar, se tiene en cuenta la materialidad que involucra la actividad (afiche para el trabajo conjunto que se replica en las fotocopias que tiene cada estudiante). Luego, se especifica el rol del docente como guía del proceso mediante preguntas que orientan la edición, que resulta del intercambio que se 
genera. Finalmente, los aspectos que se apuntan a editar están remitidos al propósito, la estructura esquemática y a los recursos enseñados en claro contraste con "las correcciones que considere pertinentes" que solo remiten a lo que los estudiantes ya saben en el Ejemplo 6. De este modo, se configura más nítidamente a la Edición como una instancia conjunta de enseñanza explícita, tal como platea la propuesta didáctica.

\section{Discusión}

Se propone, en esta sección, un análisis más abstracto de las categorías ya analizadas en función de dos dimensiones: la de la configuración del objeto de enseñanza (que abarca las categorías Géneros seleccionados y Recursos lingüísticos abordados) y la de interacción pedagógica (que engloba Guion de la Deconstrucción conjunta, Materiales diseñados para la Construcción y Guion de la Edición conjunta).

En relación con la configuración del objeto de enseñanza, se observa en SD3 cómo se logra consolidar el trabajo didáctico con textos como instancias de géneros. Así, se supera un abordaje acotado al estrato léxicogramatical para realmente enseñar cómo se construyen patrones de significado a lo largo de textos. Esto significa más genuinamente enseñar cómo usar el lenguaje de manera efectiva para leer y escribir con un propósito social vinculado a la construcción de conocimiento escolar o a la participación en problemáticas de actualidad. A su vez, en la selección de recursos lingüísticos a enseñar se evidencia que este trabajo solo cobra sentido didáctico en la medida en la que resulta relevante en un contexto de uso. Como argumentan Rose y Martin (2012:27), "esto no significa que la enseñanza del lenguaje comienza con los sistemas del lenguaje; más bien el aprendizaje del sistema del lenguaje es el punto de llegada de un extendido proceso de experimentar sus contrastes en contextos significativos" [traducción propia].

Respecto de la segunda dimensión, la interacción pedagógica, se manifiesta en el análisis diacrónico de las SD una asunción progresiva del rol docente requerido en esta propuesta didáctica. Esto implica ubicar al docente como quien guía, promueve y le da sentido al diálogo construido con sus estudiantes. Se destaca que este rol muchas veces es novedoso para los docentes en formación dado que, en muchos contextos escolares, predomina un rol docente desdibujado. Riestra (2010), en este sentido, entiende que esto es fruto de lo que denomina "posición espontaneísta frente al aprendizaje" de la lectura y la escritura, que conlleva un retraimiento de quien enseña.

Este reposicionamiento del rol docente supone un alto grado de reflexión metadidáctica que permita poner en juego una pedagogía visible (BERNSTEIN, 1990) que dé cuenta de cuáles son los propósitos que se persiguen y cuáles las actividades necesarias para realizarlos para poder garantizar que todos los estudiantes puedan participar del proceso de enseñanza-aprendizaje. En línea con esto, Rose y Martin (2012) hacen hincapié en la necesidad de la preparación efectiva para que se produzca el aprendizaje. Al respecto, se observa que en SD3 los docentes en formación plasmaron guiones y materiales que dan cuenta de que comprendieron la importancia de la preparación para la actividad como central a la tarea docente.

En síntesis, en ambas dimensiones, configuración del objeto de enseñanza e interacción pedagógica, el análisis diacrónico de las SD evidencia un alto grado de apropiación de la propuesta didáctica por parte de los docentes en formación.

Sin embargo, prospectivamente, en futuras intervenciones en la formación de formadores, se pueden pensar diversas estrategias de formación que apunten específicamente resolver algunas de las dificultades observadas en cada una de las dimensiones discutidas.

De esta manera, para ampliar el abanico de géneros discursivos abordados, y que los residentes no se limiten a aquellos de menor complejidad, se puede realizar una Deconstrucción Conjunta con SD modélicas de géneros más complejos. Esta intervención además tiene la coherencia de enseñar a planificar entendiendo a las planificaciones en sí 
como un género a enseñar, considerado metadidácticamente.

La interacción pedagógica, por su parte, es una experiencia en la que sería deseable puedan participar los residentes para ir comprendiendo gradualmente su importancia en la construcción de conocimiento conjunto. Para esto, se entiende que la demostración crítica como dispositivo de formación docente (DAVINI, 1995) puede permitir una apropiación más eficaz del rol del docente como guía de esa interacción.

\section{Conclusiones}

En este artículo, se estudiaron las secuencias didácticas (SD) elaboradas por una cohorte de docentes en formación en una propuesta didáctica basada en géneros a lo largo de un año y medio (un semestre de 2018 y el ciclo lectivo 2019).

Para analizar su evolución diacrónica se construyeron cinco categorías: Géneros seleccionados para la enseñanza, Recursos lingüísticos abordados, Guion de la Deconstrucción conjunta, Materiales diseñados para la Construcción y Guion de la Edición conjunta. En relación con la primera, se destaca que los docentes en formación tienden a recorrer el mismo trayecto de aprendizaje de géneros que propone el marco teórico para los estudiantes, avanzado gradualmente a géneros organizados por relaciones causales que ponen en juego recursos lingüísticos más abstractos. Respecto de la segunda, también se evidencia que se progresa en la enseñanza de recursos del estrato semánticodiscursivo, que supone un alcance textual más abstracto.

En cuanto a los guiones y materiales elaborados para las etapas de Deconstrucción, Construcción y Edición se observa cómo se plasma una comprensión de lo que implica un efectivo andamiaje y modelaje de cada uno de estos procesos.

Estas cinco categorías, a su vez, se integran en dos dimensiones que discutimos: la configuración del objeto de enseñanza y la interacción didáctica. En relación con la primera, se evidencia en SD3 que efectivamente el lenguaje en uso, como géneros con propósitos sociales, se constituye en el objeto de enseñanza en el aula. Respecto de la segunda, se logra plasmar "una interacción en un contexto de experiencia compartida" (MARTIN, 2000; ROSE; MARTIN, 2012), que necesariamente conlleva repensar la tarea docente activamente como guía en la construcción de conocimiento áulico.

Ambas dimensiones implican un distanciamiento crítico y un reposicionamiento respecto de una didáctica tradicional 0 de la experiencia propia de los docentes en formación como estudiantes. Por esto es que la formación docente en la propuesta centrada en géneros precisa de un abordaje recursivo, en sucesivas aproximaciones a los lineamientos teóricos y metodológicos de la propuesta. Se considera fundamental, a su vez, la instancia de las prácticas docentes como situación didáctica privilegiada que permite un nivel máximo de apropiación de la propuesta didáctica. Esto es lo que sucede en SD3, en la que efectivamente los practicantes pueden poner en juego lo planificado y experimentar no solo ese rol docente reconfigurado sino también las respuestas de los estudiantes.

Se apuesta a que los hallazgos de la investigación permitan reflexionar acerca de la formación docente (tanto inicial como continua) basada en una propuesta didáctica centrada en género $y$, de este modo, contribuir a mejorar la formación de docentes del área de Lengua/Prácticas del Lenguaje.

\section{Referências}

BERNSTEIN, Basil. Class, codes and control. Vol. 4: The structuring of pedagogic discourse. London: Routledge, 1990.

DAVINI, María Cristina. La formación en la práctica docente. Buenos Aires: Paidós, 2015.

GLASER, Barney; STRAUSS, Anselm. The discovery of grounded theory: strategies for qualitative research. Nueva York: Aldine Publishing Company, 1967. 
MARTIN, James. Mentoring semogenesis: 'genrebased' literacy pedagogy. In: CHRISTIE, Frances (Ed). Pedagogy and the Shaping of Consciousness: linguistic and social processes. London: Cassell,1999, 123-155.

MARTIN, James. Genre and language learning: A social semiotic perspective. Linguistics and Education, v.20, p. 10-21, 2009.

MARTIN, James; ROSE, David. Designing Literacy Pedagogy: scaffolding asymmetries. In: WEBSTER, Jonathan; MATTHIESSEN, Christian; HASAN, Ruqaiya (Eds.) Continuing Discourse on Language. London: Continuum, 2005, p. 251-280.

MARTIN, James; ROSE, David. Working with Discourse. Meaning beyond the clause. London: Continuum, 2007.

MARTIN, James; ROSE, David. Genre Relations. Mapping culture. London: Equinox, 2008.

MOYANO, Estela Ines. Enseñanza de la lectura y la escritura basada en la teoría de género y registro de la LSF: resultados de una investigación. Revista Signos, v. 40 n. 65 p. 573-608, 2007.

MOYANO, Estela Ines (Coord). Aprender Ciencias y Humanidades: una cuestión de lectura y escritura. Aportes para la construcción de un programa de inclusión social a través de la educación lingüística. Los Polvorines: Universidad Nacional de General Sarmiento, 2013.

RIESTRA, Dora. El papel de los géneros en las secuencias didácticas. Presentación en Segundas Jornadas Internacionales de Investigación y Prácticas en Didácticas de las Lenguas y las Literaturas, San Carlos de Bariloche, 28 y 29 de octubre, 2010.

ROSE, David; MARTIN, James. Learning to Write, Reading to Learn. Genre, Knowledge and Pedagogy in the Sydney School. UK: Equinox, 2012.

SAUTU, Ruth. Todo es teoría: objetivos y métodos de investigación. Buenos Aires: Lumiere, 2005.

BASSA FIGUEREDO, María Lorena; MOYANO, Estela Ines. Acerca de una experiencia de formación docente fundada en la didáctica de géneros: configuración del objeto de enseñanza e interacción pedagógica. Signo, Santa Cruz do Sul, v. 46, $\quad$ n. 86, may 2021. ISSN 1982-2014. Disponível em: <https://online.unisc.br/seer/index.php/signo/article/view/16062>.doi:https://doi.org/10.17058/signo.v46i86.16062. 